\title{
Development and Validation of Nomograms for Predicting the Prognosis of Triple-Negative Breast Cancer Patients Based on 379 Chinese Patients
}

This article was published in the following Dove Press journal: Cancer Management and Research

\section{Hao Shi (D) \\ Xiao-Hui Wang \\ Jun-Wei Gu \\ Gui-Long Guo}

Department of Thyroid \& Breast Surgery, The First Affiliated Hospital of Wenzhou Medical University, Wenzhou, Zhejiang,

People's Republic of China
Correspondence: Gui-Long Guo Department of Thyroid \& Breast Surgery, The First Affiliated Hospital of Wenzhou Medical University, Wenzhou, Zhejiang 325000, People's Republic of China Tel/Fax +86 577-13867709361

Email guoguilong@sina.com
Purpose: We aimed to construct universally applicable nomograms incorporating prognostic factors to predict the prognosis of patients with triple-negative breast cancer (TNBC).

Patients and methods: Clinicopathological data of 379 patients with TNBC from March 2008 to June 2014 were retrospectively collected and analyzed. The endpoints were disease-free survival (DFS) and overall survival (OS). Patients were randomly divided into a training group and an independent validation group. In the training group, the prognostic factors were screened to develop nomograms. C-index and calibration curves were used to evaluate the predictive accuracy and discriminative ability of nomograms in both groups. The accuracy of the nomograms was also compared with the traditional American Joint Committee on Cancer Tumor-Node-Metastasis anatomical stage (8th edition).

Results: Four prognostic factors (albumin-to-globulin ratio, neutrophil-to-lymphocyte ratio, positive lymph nodes, and tumor size) were used to construct the nomogram of DFS. In addition to the aforementioned factors, age was taken into account in the construction of the OS nomogram. The C-index of the DFS nomogram in the training and validation groups was 0.71 (95\% confidence interval [CI]: 0.64-0.77) and 0.69 (95\% CI: 0.58-0.79), respectively; the C-index of the OS nomogram was 0.77 (95\% CI: $0.70-0.84)$ and 0.74 (95\% CI: 0.62 0.86 ), respectively. This suggests that the nomograms had high accuracy. Moreover, calibration curves showed good consistencies in both groups. Our models showed superiority in predicting accuracy compared with the AJCC TNM staging system. Furthermore, two web pages of the nomograms were produced: DFS: https://sh-skipper.shinyapps.io/TNBC1/; OS: https://sh-skipper.shinyapps.io/TNBC2/.

Conclusion: These predictive models are simple and easy to use, particularly the web versions. They have certain clinical value in predicting the prognosis of patients with TNBC. They can assist doctors in identifying patients at different prognostic risks and strengthen the treatment or follow-up accordingly.

Keywords: triple negative breast cancer, prognosis, prediction, nomograms

\section{Introduction}

Breast cancer has become the most common malignant tumor in females worldwide, and the incidence of breast cancer in China is increasing annually. Although the mortality rate of breast cancer has declined in recent years owing to advancements in cancer treatment, the disease remains the main cause of cancer-related death in females. ${ }^{1,2}$ Triple-negative breast cancer (TNBC) refers to breast cancer with negative estrogen receptor, negative progesterone receptor, and negative human epidermal growth factor receptor-2 (HER2) status. It is a special subtype 
of breast cancer, prone to local recurrence and distant metastasis, accounting for approximately $15-20 \%$ of all invasive breast cancer cases. ${ }^{3-6}$ Owing to its particular malignant behavior, it is meaningful to stratify patients with TNBC based on their individual characteristics to better assess prognosis.

The nomogram can integrate several important factors to predict a specific endpoint in a graphical representation, indicating that this approach can predict the prognosis of patients according to their individual data. This is of profound significance in clinical practice, especially for the prognosis of patients with cancer. ${ }^{7}$ At present, there are few nomograms for TNBC.

Therefore, we retrospectively analyzed the clinical and pathological data of 379 patients with TNBC to construct widely applicable prognostic nomograms in this setting.

\section{Patients and Methods}

\section{Patient Selection and General Information}

The clinicopathological data of patients who were diagnosed with TNBC and underwent surgery at the First Affiliated Hospital of Wenzhou Medical University from March 2008 to June 2014 were collected. Inclusion criteria were: (1) female patients; (2) patients with primary TNBC; (3) unilateral breast cancer; (4) no other diagnosis of cancer; and (5) no recent inflammatory disease. Exclusion criteria were: (1) carcinoma in situ; (2) inflammatory breast cancer or distant metastasis; (3) history of acute or chronic liver disease or kidney disease; and (4) combination with serious heart and lung diseases. The criteria for determining TNBC were: estrogen receptor and progesterone receptor negative status, with reference to immunohistochemical staining positive cells $<1 \%$. HER2 $(+++)$ denoted overexpression of HER2. HER2 $(-)$ and $(+)$ were considered HER2 negative, and HER2 $(++)$ required further evaluation through fluorescence in situ hybridization to determine the presence of overexpression. The positive result of fluorescence in situ hybridization was judged to be HER2 positive, whereas the opposite was judged to be negative. Finally, a total of 379 patients were enrolled, and all patients underwent a wellestablished preoperative assessment, such as blood biochemical examination, electrocardiography, breast ultrasound, etc. Postoperative adjuvant therapy was performed routinely according to the Chinese treatment guidelines at that time. Relevant clinical data were collected as shown in
Table I Clinicopathological Characteristics of the Training and Validation Groups

\begin{tabular}{|c|c|c|c|}
\hline Characteristic & $\begin{array}{l}\text { Training Group } \\
(\mathrm{N}=255) \text { No. (\%) }\end{array}$ & $\begin{array}{l}\text { Validation } \\
\text { Group } \\
(\mathrm{N}=124) \text { No. (\%) }\end{array}$ & p-value \\
\hline $\begin{array}{l}\text { Age (years) } \\
\quad \leq 60 \\
>60\end{array}$ & $\begin{array}{l}212(83.1) \\
43(16.9)\end{array}$ & $\begin{array}{l}103(83.1) \\
21(16.9)\end{array}$ & 0.986 \\
\hline $\begin{array}{c}\text { BMI }\left(\mathrm{kg} / \mathrm{m}^{2}\right) \\
\quad<18.5 \\
18.5-24 \\
>24\end{array}$ & $\begin{array}{l}\text { II (4.3) } \\
179(70.2) \\
65(25.5)\end{array}$ & $\begin{array}{l}7(5.7) \\
81(65.3) \\
36(29.0)\end{array}$ & 0.641 \\
\hline $\begin{array}{l}\text { Menstrual status } \\
\text { Premenopausal } \\
\text { Postmenopausal }\end{array}$ & $\begin{array}{l}134(52.5) \\
121(47.5)\end{array}$ & $\begin{array}{l}58(46.8) \\
66(53.2)\end{array}$ & 0.291 \\
\hline $\begin{array}{l}\text { Neutrophils } \\
\begin{array}{l}\left(\times 10^{9} / \mathrm{L}\right) \\
\leq 3.6 \\
>3.6\end{array}\end{array}$ & $\begin{array}{l}|2|(47.5) \\
134(52.5)\end{array}$ & $\begin{array}{l}52(41.9) \\
72(58.1)\end{array}$ & 0.312 \\
\hline $\begin{array}{l}\text { Lymphocytes } \\
\begin{array}{l}\left(\times 10^{9} / \mathrm{L}\right) \\
\quad<1.8 \\
\geq 1.8\end{array}\end{array}$ & $\begin{array}{l}123(48.2) \\
132(51.8)\end{array}$ & $\begin{array}{l}70(56.4) \\
54(43.6)\end{array}$ & 0.133 \\
\hline $\begin{array}{l}\text { Platelets }\left(\times 10^{9} / \mathrm{L}\right) \\
\quad \leq 210 \\
>210\end{array}$ & $\begin{array}{l}125(49.0) \\
130(51.0)\end{array}$ & $\begin{array}{l}55(44.4) \\
69(55.6)\end{array}$ & 0.394 \\
\hline $\begin{array}{l}\text { Total protein }(\mathrm{g} / \mathrm{L}) \\
\quad \leq 74 \\
>74\end{array}$ & $\begin{array}{l}123(48.2) \\
132(51.8)\end{array}$ & $\begin{array}{l}63(50.8) \\
61(49.2)\end{array}$ & 0.639 \\
\hline $\begin{array}{l}\text { Albumin }(g / L) \\
\quad<45 \\
\geq 45\end{array}$ & $\begin{array}{l}120(47.1) \\
135(52.9)\end{array}$ & $\begin{array}{l}58(46.8) \\
66(53.2)\end{array}$ & 0.958 \\
\hline $\begin{array}{l}\text { AGR } \\
\quad \leq 1.3 \\
>1.3\end{array}$ & $\begin{array}{l}68(26.7) \\
187(73.3)\end{array}$ & $\begin{array}{l}27(21.8) \\
97(78.2)\end{array}$ & 0.302 \\
\hline $\begin{array}{l}\text { PLR } \\
\qquad 145 \\
\geq 145\end{array}$ & $\begin{array}{l}189(74.1) \\
66(25.9)\end{array}$ & $\begin{array}{l}84(67.8) \\
40(32.2)\end{array}$ & 0.194 \\
\hline $\begin{array}{l}\text { NLR } \\
\quad \leq 3.1 \\
>3.1\end{array}$ & $\begin{array}{l}229(89.8) \\
26(10.2)\end{array}$ & $\begin{array}{l}104(83.9) \\
20(16.1)\end{array}$ & 0.097 \\
\hline $\begin{array}{l}\text { SII } \\
\qquad 500 \\
>500\end{array}$ & $\begin{array}{l}163(63.9) \\
92(36.1)\end{array}$ & $\begin{array}{l}69(55.6) \\
55(44.4)\end{array}$ & 0.121 \\
\hline $\begin{array}{l}\text { Surgery type } \\
\text { Mastectomy } \\
\text { BCS }\end{array}$ & $\begin{array}{l}204(80.0) \\
51(20.0)\end{array}$ & $\begin{array}{l}106(85.5) \\
18(14.5)\end{array}$ & 0.205 \\
\hline
\end{tabular}

(Continued) 
Table I (Continued).

\begin{tabular}{|c|c|c|c|}
\hline Characteristic & $\begin{array}{l}\text { Training Group } \\
(\mathrm{N}=255) \text { No. (\%) }\end{array}$ & $\begin{array}{l}\text { Validation } \\
\text { Group } \\
(\mathrm{N}=124) \text { No. (\%) }\end{array}$ & p-value \\
\hline $\begin{array}{l}\text { Histopathological } \\
\text { type } \\
\text { IDC } \\
\text { Others }\end{array}$ & $\begin{array}{l}219(85.9) \\
36(14.1)\end{array}$ & $\begin{array}{l}105(84.7) \\
19(15.3)\end{array}$ & 0.755 \\
\hline $\begin{array}{l}\text { Tumor size }(\mathrm{cm}) \\
\quad \leq 2 \\
2-5 \\
>5\end{array}$ & $\begin{array}{l}122(47.8) \\
126(49.4) \\
7(2.8)\end{array}$ & $\begin{array}{l}70(56.5) \\
49(39.5) \\
5(4.0)\end{array}$ & 0.180 \\
\hline $\begin{array}{l}\text { Positive lymph } \\
\text { nodes } \\
0 \\
\text { I-3 } \\
\geq 4\end{array}$ & $\begin{array}{l}160(62.7) \\
62(24.3) \\
33(13.0)\end{array}$ & $\begin{array}{l}81(65.3) \\
25(20.2) \\
18(14.5)\end{array}$ & 0.648 \\
\hline $\begin{array}{l}\mathrm{Ki}-67^{\mathrm{a}}(\%) \\
\quad \leq 30 \\
>30\end{array}$ & $\begin{array}{l}76(29.8) \\
166(65.1)\end{array}$ & $\begin{array}{l}40(32.3) \\
78(62.9)\end{array}$ & 0.635 \\
\hline
\end{tabular}

Notes: ${ }^{\mathrm{a}} \mathrm{Ki}-67$ had missing data.

Abbreviations: AGR, albumin-to-globulin ratio; PLR, platelet-to-lymphocyte ratio; NLR, neutrophil-to-lymphocyte ratio; SIl, systemic immune inflammation index, defined as (platelets $\times$ neutrophils)/lymphocytes; BCS, breast-conserving surgery; IDC, invasive ductal carcinoma.

Table 1, including age, menstrual status, the latest blood biochemical indicators prior to surgical operation, tumor size, lymph node status, and other factors.

\section{Follow-Up and Endpoints}

Follow-up data were obtained via telephone interviews and outpatient follow-up after operation. In this study, local recurrence and distant metastasis are collectively referred to as breast cancer recurrence. Disease-free survival (DFS) time is defined as the time from the surgery to recurrence (clinical, radiological or pathological evidence), death, or last follow-up. Overall survival (OS) time is defined as the time form the operation to death or last follow-up. The deadline for follow-up was June 2019.

\section{Statistical Analysis}

Statistical analysis was performed using the SPSS version 22.0 software package (IBM Corp., Armonk, NY, USA). The chi-squared test was used to compare the rates. Univariate comparisons of survival data were performed using the Kaplan-Meier method and Cox univariate analysis. Based on the results of the univariate analysis $(\mathrm{p}<0.05)$ and combined with clinical important factors, further multivariate analysis using the Cox risk regression model with backward elimination was performed. Following the multivariate analysis, variables with a p-value $<0.1$ were selected for developing the nomograms. The nomograms were constructed using the R software (version R-3.5.3 https://www.r-project.org/). $\mathrm{P}$-values were two-sided, and $\mathrm{p}<0.05$ denoted statistical significance. The cutoff values of the variables were determined based on clinical, relevant studies, and the X-tile software (Yale University, New Haven,CT, USA version 3.6.1).

\section{Results}

\section{Patient Information}

The median follow-up time for the 379 patients with TNBC was 71 months (range: 3-133 months). The median age was 49 years (range: 25-84 years). Approximately half of the patients were menopausal. Among the pathological types, invasive ductal carcinoma accounted for the majority $(85.5 \%)$, while the rest were invasive lobular carcinoma, medullary carcinoma, and other types. A total of 138 patients had lymph node metastasis, accounting for $36.4 \%$ of all patients. Notably, 69 patients underwent breast-conserving surgery. The rate of breast-conserving surgery was approximately $18.2 \%$. Tumors with a size $\geq 2 \mathrm{~cm}$ accounted for approximately half of the cases $(49.3 \%)$. There were 84 patients who developed recurrence, and 63 patients expired. The DFS and OS rates were $77.8 \%$ and $83.4 \%$, respectively. The $\mathrm{R}$ software was used to randomly divide all 379 patients (67\%, seed 60) into two groups: the training group (255 patients) and the validation group (124 patients). The clinicopathological features of the two groups are presented in Table 1. In the training group, 52 patients relapsed and 39 patients expired. In the validation group, 32 patients relapsed and 24 patients expired. There was no statistical difference between the two groups in terms of clinicopathological characteristics (Table 1).

\section{Prognostic Factors of DFS and OS}

The univariate analysis showed that age $(\mathrm{p}=0.024)$, albumin-to-globulin ratio ( $\mathrm{AGR} ; \mathrm{p}=0.002$ ), tumor size, and lymph node metastasis were related to prognosis. Although the neutrophil-to-lymphocyte ratio (NLR) did not show statistical significance, numerous studies have 
Table 2 Univariate and Multivariate Analyses of DFS in the Training Group

\begin{tabular}{|c|c|c|c|c|}
\hline \multirow[t]{2}{*}{ Characteristic } & \multicolumn{2}{|c|}{ Univariate Analysis of DFS } & \multicolumn{2}{|c|}{ Multivariate Analysis of DFS } \\
\hline & HR (95\% Cl) & p-value & HR $(95 \% \mathrm{Cl})$ & p-value \\
\hline \multicolumn{5}{|l|}{ Age (years) } \\
\hline$\leq 60$ & I & & 1 & \\
\hline$>60$ & $2.031(1.099-3.755)$ & 0.024 & $1.660(0.868-3.174)$ & 0.125 \\
\hline \multicolumn{5}{|l|}{ AGR } \\
\hline$\leq 1.3$ & 1 & & 1 & \\
\hline$>1.3$ & $0.414(0.238-0.722)$ & 0.002 & $0.463(0.264-0.812)$ & 0.007 \\
\hline \multicolumn{5}{|l|}{$N L R^{a}$} \\
\hline$\leq 3.1$ & 1 & & 1 & \\
\hline$>3.1$ & $1.870(0.911-3.837)$ & 0.088 & $2.117(1.026-4.367)$ & 0.042 \\
\hline \multicolumn{5}{|l|}{ Tumor size $(\mathrm{cm})$} \\
\hline$\leq 2$ & I & & 1 & \\
\hline $2-5$ & $\mathrm{I} .422(0.797-2.537)$ & 0.234 & $\mathrm{I} .426(0.79 \mathrm{I}-2.570)$ & 0.237 \\
\hline$>5$ & $6.937(2.592-18.567)$ & 0.000 & $2.644(0.877-7.975)$ & 0.084 \\
\hline \multicolumn{5}{|l|}{ Positive lymph nodes } \\
\hline 0 & 1 & & 1 & \\
\hline $1-3$ & $1.687(0.868-3.246)$ & 0.124 & $1.763(0.909-3.420)$ & 0.093 \\
\hline$\geq 4$ & $3.647(1.885-7.055)$ & 0.000 & $3.341(1.709-6.532)$ & 0.000 \\
\hline BMI $\left(\mathrm{kg} / \mathrm{m}^{2}\right)$ & - & 0.893 & & \\
\hline Menstrual status & $1.56 \mid(0.900-2.707)$ & 0.113 & & \\
\hline Neutrophils $\left(\times 10^{9} / \mathrm{L}\right)$ & $1.200(0.694-2.075)$ & 0.514 & & \\
\hline Lymphocytes $\left(\times 10^{9} / \mathrm{L}\right)$ & $0.608(0.349-1.057)$ & 0.078 & & \\
\hline Platelets $\left(\times 10^{9} / \mathrm{L}\right)$ & $1.150(0.666-1.984)$ & 0.617 & & \\
\hline Total protein $(g / L)$ & I. $174(0.679-2.029)$ & 0.567 & & \\
\hline Albumin (g/L) & $0.604(0.348-1.047)$ & 0.072 & & \\
\hline PLR & $1.709(0.972-3.006)$ & 0.063 & & \\
\hline SII & $1.416(0.819-2.448)$ & 0.213 & & \\
\hline Surgery type & $1.003(0.515-1.951)$ & 0.993 & & \\
\hline Histopathological type & $0.904(0.408-2.005)$ & 0.804 & & \\
\hline $\mathrm{Ki}-67(\%)$ & $0.889(0.495-1.597)$ & 0.694 & & \\
\hline
\end{tabular}

Notes: ${ }^{a}$ Considering the clinical importance of NLR, it was also included in the multivariate analysis.

Abbreviations: DFS, disease-free survival; HR, hazard ratio; Cl, confidence interval; AGR, albumin-to-globulin ratio; NLR, neutrophil-to-lymphocyte ratio; BMI, body mass index; PLR, platelet-to-lymphocyte; SII, systemic immune inflammation index, defined as (platelets×neutrophils)/lymphocytes.

confirmed its clinical importance; hence, we incorporated this factor into the multivariate model. The final Cox multivariate analysis showed that AGR (hazards ratio $[\mathrm{HR}]=0.463,95 \%$ confidence interval $[\mathrm{CI}]: 0.264-0.812$, $\mathrm{p}=0.007), \quad \mathrm{NLR} \quad(\mathrm{HR}=2.117, \quad 95 \% \quad \mathrm{CI}: \quad 1.026-4.367$, $\mathrm{p}=0.042$ ), and lymph node status were independent prognostic factors of DFS (Table 2). Similar to DFS, the univariate analysis of OS showed that age $(p=0.008)$, AGR $(\mathrm{p}=0.001)$, tumor size, lymph node status, and NLR $(p=0.033)$ were associated with prognosis. Based on the above variables, the multivariate analysis showed that AGR (HR=0.471, 95\% CI: 0.239-0.930, p=0.030), NLR $(\mathrm{HR}=3.072$, 95\% CI: 1.389-6.796, $\mathrm{p}=0.006)$, and lymph node metastasis were independent prognostic factors of OS (Table 3). The survival curves of subgroups with different AGR or NLR are shown in Figure 1. 
Table 3 Univariate and Multivariate Analyses of OS in the Training Group

\begin{tabular}{|c|c|c|c|c|}
\hline \multirow[t]{2}{*}{ Characteristics } & \multicolumn{2}{|c|}{ Univariate Analysis of OS } & \multicolumn{2}{|c|}{ Multivariate Analysis of OS } \\
\hline & HR (95\% Cl) & p-value & HR (95\% Cl) & p-value \\
\hline \multicolumn{5}{|l|}{ Age (years) } \\
\hline$\leq 60$ & I & & 1 & \\
\hline$>60$ & $2.500(1.264-4.945)$ & 0.008 & $2.044(0.987-4.230)$ & 0.054 \\
\hline \multicolumn{5}{|l|}{ AGR } \\
\hline$\leq 1.3$ & I & & 1 & \\
\hline$>1.3$ & $0.349(0.184-0.662)$ & 0.001 & $0.471(0.239-0.930)$ & 0.030 \\
\hline \multicolumn{5}{|l|}{ NLR } \\
\hline$\leq 3.1$ & I & & 1 & \\
\hline$>3.1$ & $2.352(1.068-5.059)$ & 0.033 & $3.072(1.389-6.796)$ & 0.006 \\
\hline \multicolumn{5}{|l|}{ Tumor size $(\mathrm{cm})$} \\
\hline$\leq 2$ & 1 & & 1 & \\
\hline $2-5$ & $1.658(0.829-3.314)$ & 0.153 & $1.670(0.824-3.386)$ & 0.155 \\
\hline$>5$ & $9.943(3.529-28.016)$ & 0.000 & $3.223(1.008-10.305)$ & 0.048 \\
\hline \multicolumn{5}{|l|}{ Positive lymph nodes } \\
\hline 0 & 1 & & 1 & \\
\hline $1-3$ & I.77I (0.803-3.904) & 0.157 & $2.066(0.928-4.602)$ & 0.076 \\
\hline$\geq 4$ & $5.194(2.496-10.810)$ & 0.000 & $4.731(2.227-10.048)$ & 0.000 \\
\hline BMI $\left(\mathrm{kg} / \mathrm{m}^{2}\right)$ & - & 0.430 & & \\
\hline Menstrual status & $1.651(0.872-3.126)$ & 0.123 & & \\
\hline Neutrophils $\left(\times 10^{9} / \mathrm{L}\right)$ & $1.723(0.895-3.316)$ & 0.103 & & \\
\hline Lymphocytes $\left(\times 10^{9} / \mathrm{L}\right)$ & $0.56 \mid(0.294-1.069)$ & 0.079 & & \\
\hline Platelets $\left(\times 10^{\wedge} 9 / \mathrm{L}\right)$ & $1.296(0.688-2.443)$ & 0.422 & & \\
\hline Total protein $(\mathrm{g} / \mathrm{L})$ & $1.081(0.576-2.031)$ & 0.808 & & \\
\hline Albumin $(\mathrm{g} / \mathrm{L})$ & $0.57 \mid(0.30 I-I .08 I)$ & 0.085 & & \\
\hline PLR & $1.872(0.982-3.569)$ & 0.057 & & \\
\hline SII & 1.947 (1.039-3.649) & 0.038 & & \\
\hline Surgery type & $0.662(0.277-1.58 I)$ & 0.353 & & \\
\hline Histopathological type & $0.872(0.34 I-2.230)$ & 0.775 & & \\
\hline $\mathrm{Ki}-67$ (\%) & $1.004(0.506-1.992)$ & 0.991 & & \\
\hline
\end{tabular}

Abbreviations: OS, overall survival; $\mathrm{HR}$, hazard ratio; $\mathrm{Cl}$, confidence interval; AGR, albumin-to-globulin ratio; NLR, neutrophil-to-lymphocyte ratio; BMI, body mass index; PLR, platelet-to-lymphocyte ratio; SIl, systemic immune inflammation index defined as (platelets $\times$ neutrophils)/lymphocytes.

\section{Development of Prediction Models}

The R software was used to develop DFS and OS nomograms for the prediction of patient prognosis. Several variables had no significance in the multivariate analysis; however, they were included in the nomogram according to the exclusion criterion of 0.1 . The nomogram of DFS is shown in Figure 2. The total scores of each prognostic index were added to obtain the corresponding prognosis.
For example, a 50-year-old patient with TNBC has an AGR of 1.3, NLR of 2, a tumor with a maximum diameter of $2.5 \mathrm{~cm}$, and two positive lymph nodes. The corresponding AGR and NLR scores for this patient were 68 and 0 , respectively. The score of tumor size was 29 , and the score of the lymph node condition was 48 . Thus, the aggregate score was $68+0+29+48=145$. The corresponding 3 -year and 5-year DFS rates were approximately $75 \%$ and $64 \%$, 

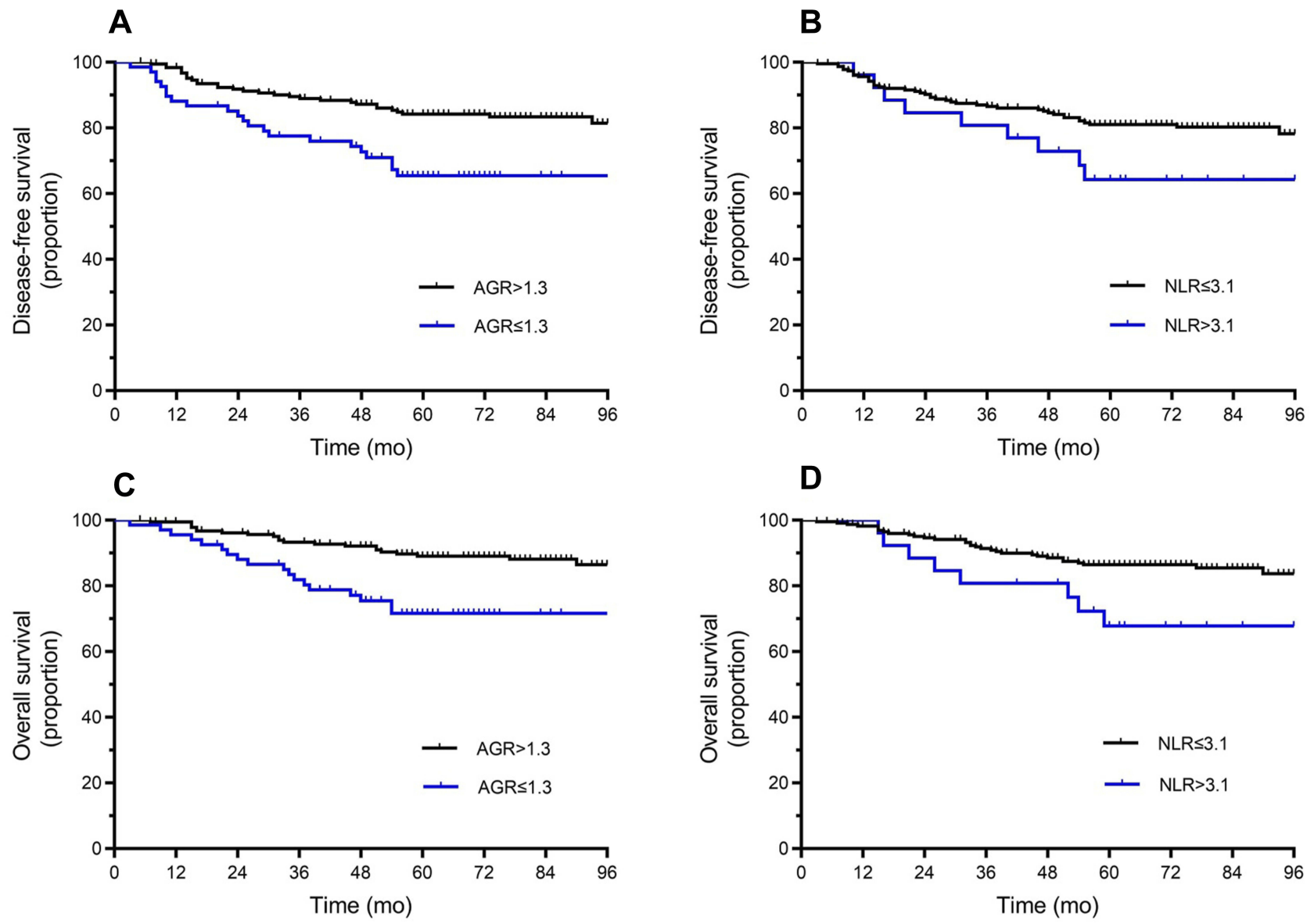

Figure I Kaplan-Meier survival curves in subgroups with different AGR or NLR of the training group. (A, B) Disease-free survival. (C, D) Overall survival. Abbreviations: AGR, albumin-to-globulin ratio; NLR, neutrophil-to-lymphocyte ratio.

respectively. The OS nomogram is shown in Figure 3. The total score was generated to predict the corresponding prognosis in the same manner as for DFS. For this patient, the corresponding scores were 0 for age, 49 for AGR, 49 for NLR, 39 for tumor size, and 46 for lymph nodes; hence the total score was $0+49+0+39+46=134$. The predictive rates of 3- and 5-year OS for were approximately $85 \%$ and $76 \%$, respectively.

\section{Evaluation of the Prediction Models}

In the training group, the C-index of DFS was $0.71(95 \%$ CI: $0.64-0.77$ ), while that of OS was 0.77 (95\% CI: 0 .70-0.84). The calibration curves revealed great consistencies, as shown in Figure 4. We used the survival data of 124 patients with TNBC in the validation group to further verify whether the predictive models can differentiate the prognosis of different patients with TNBC. In the validation group, the C-index of DFS was 0.69 (95\% CI: 0.58 0.79), while that of OS was 0.74 (95\% CI: $0.62-0.86)$. The calibration curves are shown in Figure 5. There were good consistencies between the predicted values and the actual observed situation.

We also compared our models with the traditional AJCC TNM anatomical staging system (8th edition) using the overall data. Figure $6 \mathrm{~A}$ and $\mathrm{B}$ illustrate the results of the time-dependent receiver operating characteristic curve analysis. The findings indicated that, unlike the TNM staging system, our model had good stability over time. The difference in the area under the curve between the prediction models and the TNM staging system can be observed from Figure 6C and D. There were no significant differences in the area under the curve in the short term. However, with the extension of time, the advantages of our prediction models became obvious. The 5-year receiver operating characteristic curves are shown in Figure 7. According to these, our models showed superiority in predicting accuracy compared with the traditional TNM staging system. 
Points

$\begin{array}{lllllllllll}0 & 10 & 20 & 30 & 40 & 50 & 60 & 70 & 80 & 90 & 100\end{array}$

AGR

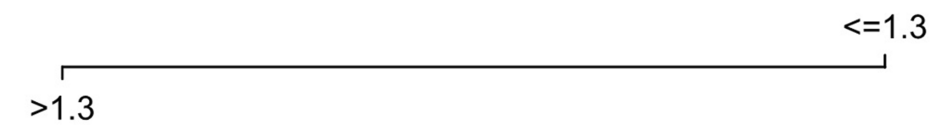

NLR

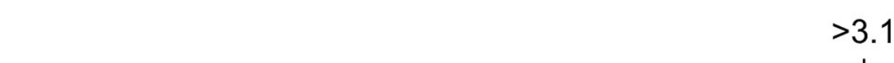

$$
<=3.1
$$

Tumor.size

$$
2-5 \mathrm{~cm}
$$

Positive.lymph.nodes

$$
<=2 \mathrm{~cm}
$$

\section{Total Points}

\section{3-years DFS rate}

\section{5-years DFS rate}

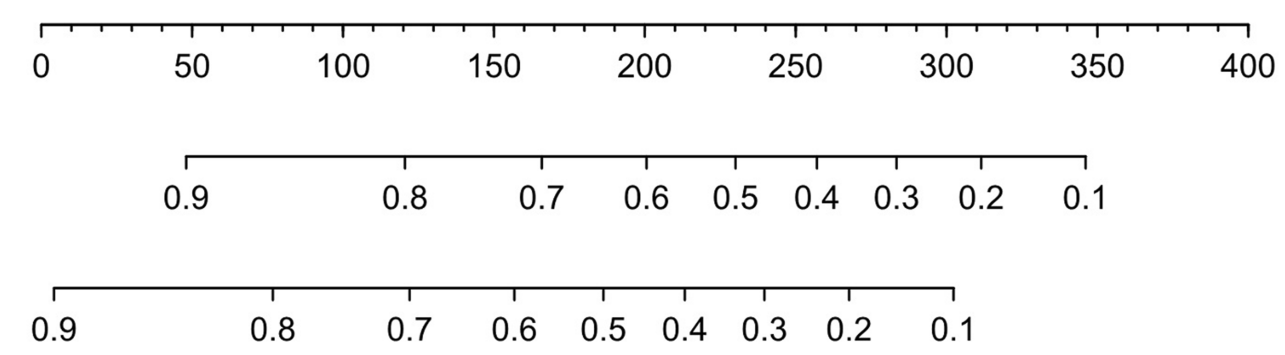

Figure 2 Nomogram for the prediction of DFS in triple-negative breast cancer.

Abbreviations: DFS, disease-free survival; AGR, albumin-to-globulin ratio; NLR, neutrophil-to-lymphocyte ratio.

Based on the nomograms, we further developed the following two simple web pages which contain more detailed prognostic information:

DFS: https://sh-skipper.shinyapps.io/TNBC1/; OS: https://sh-skipper.shinyapps.io/TNBC2/.

\section{Discussion}

Strong invasiveness and poor prognosis are the characteristics of TNBC which is more prone to distant metastasis than other types, such as the lung and brain. ${ }^{3,8}$ In our data, the lung was the most common site of metastasis (29/84 patients) followed by bone metastasis (16/ 84 patients). The recurrence of TNBC peaked within 3 years after treatment and decreased after 5 years. ${ }^{9,10}$ Therefore, we mainly combined clinical and laboratoryrelated prognostic factors for the construction of a 3-year and 5-year nomogram to better predict the prognosis of patients with TNBC. Through univariate and multivariate analyses, we found that age, lymph node status, and tumor size were associated with prognosis, similar to previous studies investigating risk factors related to TNBC in China. ${ }^{11,12}$
Tumor-related inflammation is an important component of the tumor microenvironment and immune inflammation plays an important role in the occurrence and development of tumors. ${ }^{13}$ TNBC is closely related to tumor immune inflammation. Inflammation and the immune status affect the occurrence and development of TNBC. ${ }^{14}$ Inflammation-immune circulating cells (eg, neutrophils, lymphocytes, platelets, etc) can influence the proliferation, invasion, and metastasis of various solid tumors, including breast cancer. The above cells can be detected through routine blood testing as markers of inflammation. Preoperative NLR, as a reliable indicator of systemic inflammation, has been increasingly associated with the prognosis of diverse solid tumors, including TNBC. ${ }^{15}$ A meta-analysis of 40,559 patients with solid tumors showed that an NLR $>4.00$ significantly increased the adverse prognosis of solid tumors, which further confirmed the aforementioned hypothesis. ${ }^{16}$ Therefore, in our study, we used the NLR as a candidate for constructing the model. Similar to previous studies, we chose 3.1 as the cutoff value. ${ }^{17,18}$ For DFS, the univariate analysis of NLR did not show statistical significance. However, considering 


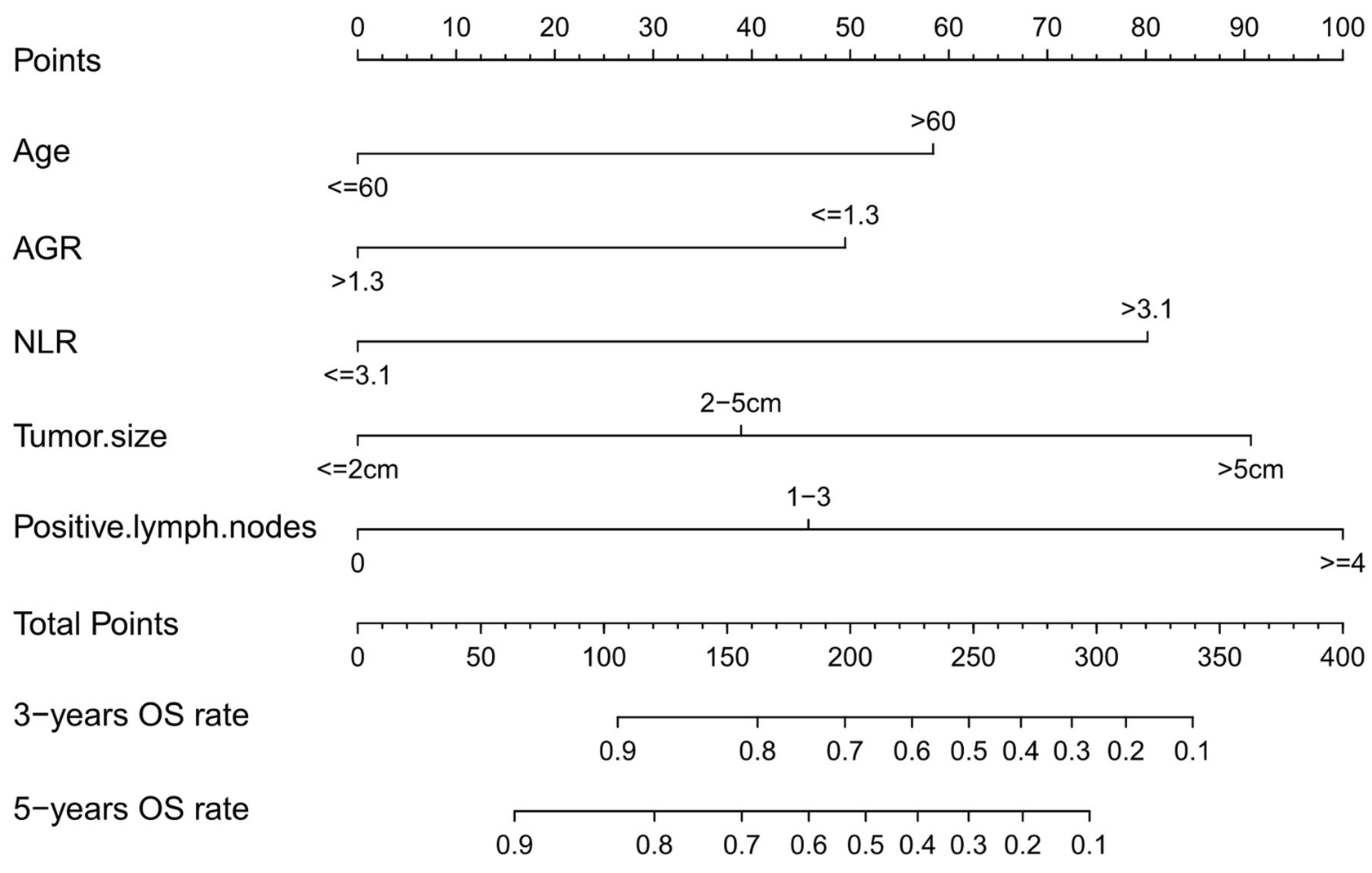

Figure 3 Nomogram for the prediction of OS in triple-negative breast cancer.

Abbreviations: OS, overall survival; AGR, albumin-to-globulin ratio; NLR, neutrophil-to-lymphocyte ratio.

the clinical importance of NLR, it was also included in the multivariate analysis. The final multivariate analysis showed that NLR was an independent predictor of DFS in patients with TNBC. Regarding OS, both univariate and multivariate analyses showed statistical significance. Consistent with previous studies, our study suggests that NLR is an independent prognostic factor for TNBC. The systemic immune inflammation index (SII) is based on peripheral neutrophils, lymphocytes, and platelet counts. It is defined as (platelets $\times$ neutrophils)/lymphocytes, and is a potential indicator of host inflammation and immune status. Research studies were conducted on various cancers (eg, liver cancer, colorectal cancer, pancreatic cancer, etc), all showing a good predictive effect. ${ }^{19-22}$ Although there were few studies on TNBC, Wang et al showed its prognostic significance. ${ }^{23}$ However, in our data, there was no statistical significance for DFS. The univariate analysis of OS showed that SII had a tendency to be a prognostic indicator; however, the bivariate correlation analysis showed that it was correlated with NLR, and its calculation was not as simple as that for NLR. Hence, it was not included in the final model. The platelet-to-lymphocyte ratio can comprehensively reflect the dynamic changes between platelets and lymphocytes in the peripheral blood circulation. Studies have confirmed that the pretreatment platelet-to-lymphocyte ratio is related to the prognosis of a variety of malignant tumors, including hepatocellular carcinoma, colorectal cancer, breast cancer, etc. ${ }^{24-26}$ Unfortunately, no prognostic value was found in our study.

Albumin and globulin are two major components of serum protein, which play important roles in the inflammatory process. AGR is a common index in blood biochemical examination. Owing to its simplicity, costeffectiveness, and practicability, AGR can be used as a disease-monitoring and prognostic indicator for a variety of cancers, including breast cancer. ${ }^{27-29}$ In our study, using the X-tile software with an optimal cutoff value of 1.3, we showed that the pre-treatment AGR was an independent predictor of prognosis in patients with TNBC. The prognosis of patients with a high AGR is better, whereas that of patients with a low AGR is poor. This suggests that, during the treatment of patients with TNBC, active nutritional support, correction of 
A

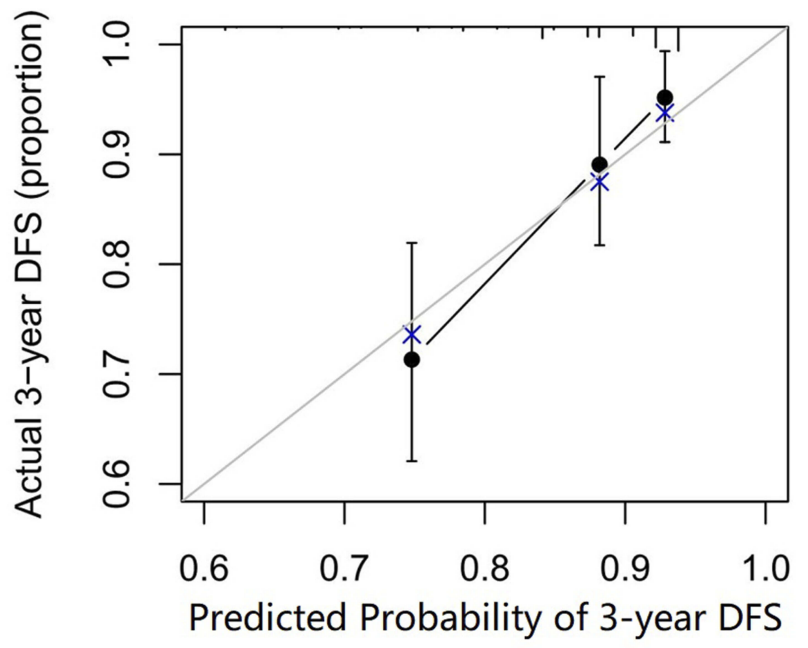

C

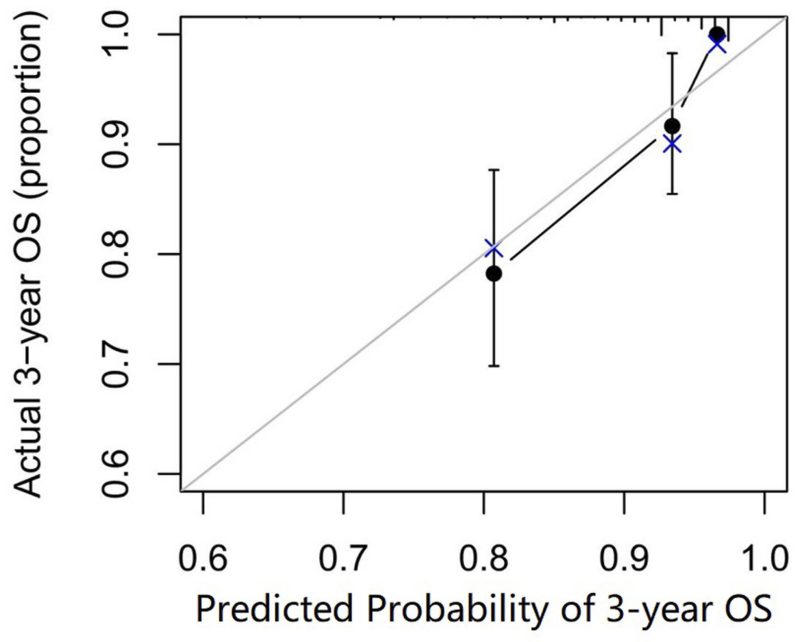

B

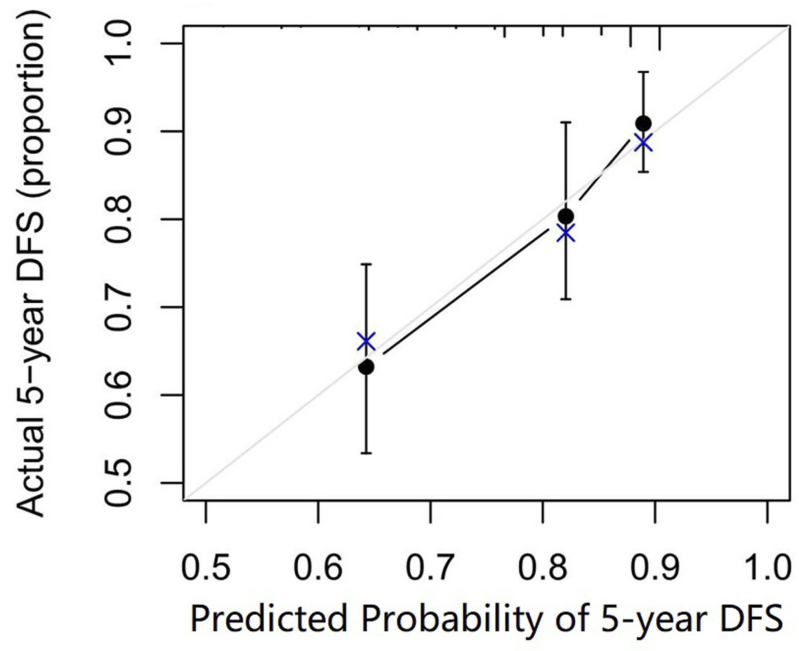

D

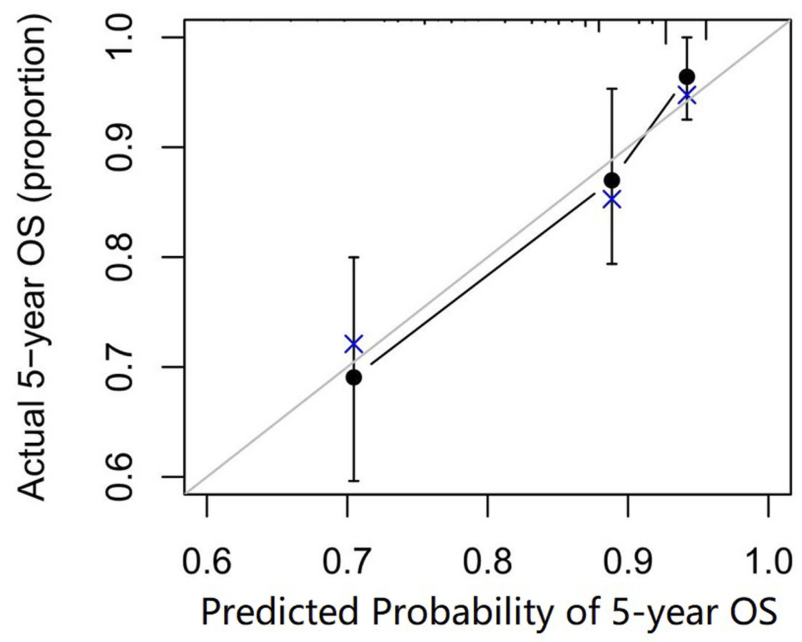

Figure 4 Calibration curves for predicting the OS and DFS for patients with triple-negative breast cancer in the training group at (A, C) 3 years and (B, D) 5 years. Nomogram-predicted probability is plotted on the $\mathrm{x}$-axis and the actual survival is plotted on the $y$-axis.

Abbreviations: DFS, disease-free survival; OS, overall survival.

hypoproteinemia, and other measures are necessary to improve the status of the AGR and potentially the prognosis to some extent.

In addition to the factors included in our study, there are numerous predictors for the prognosis of TNBC. Herein, we discussed the potential factors which may enhance the performance of nomograms. Serum tumor markers are relatively convenient and cheap, and their importance cannot be ignored. Previous studies showed that the levels of carcinoembryonic antigen (CEA) and cancer antigen 15-3 (CA15-3) are independent prognostic factors for breast cancer. Dai et $\mathrm{al}^{30}$ collected and analyzed the CA15-3, CEA, and other factors of 247 patients with TNBC prior to treatment. Their results showed that high CA15-3 and CEA levels were associated with poor prognosis of TNBC. Firstly, we included these factors in our analysis. Unfortunately, we found that the levels of CEA and CA15-3 were not determined in many of our earlier patients. Adams et $\mathrm{al}^{31}$ collected 481 cases of patients with TNBC in two prospective randomized controlled clinical trials (ECoG 2197 and ECoG 1199). They determined the density of intraepithelial tumor infiltrating lymphocytes (TILs) and stromal TILs in tumor samples. The results showed that TIL was an independent prognostic marker and a powerful prognostic factor for TNBC. Dieci et $\mathrm{al}^{32}$ also confirmed the prognostic role of TIL in 816 patients with TNBC (781 of whom were evaluable). 
A

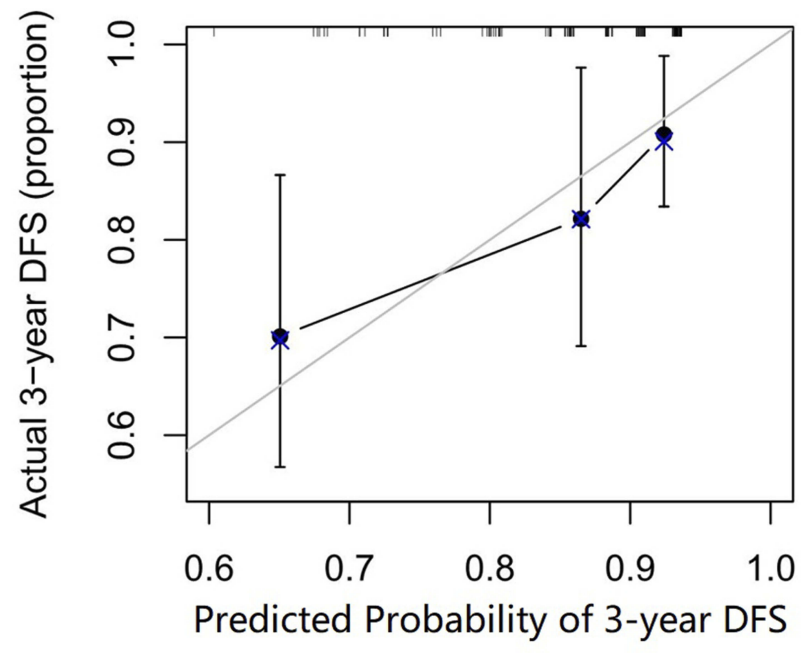

C

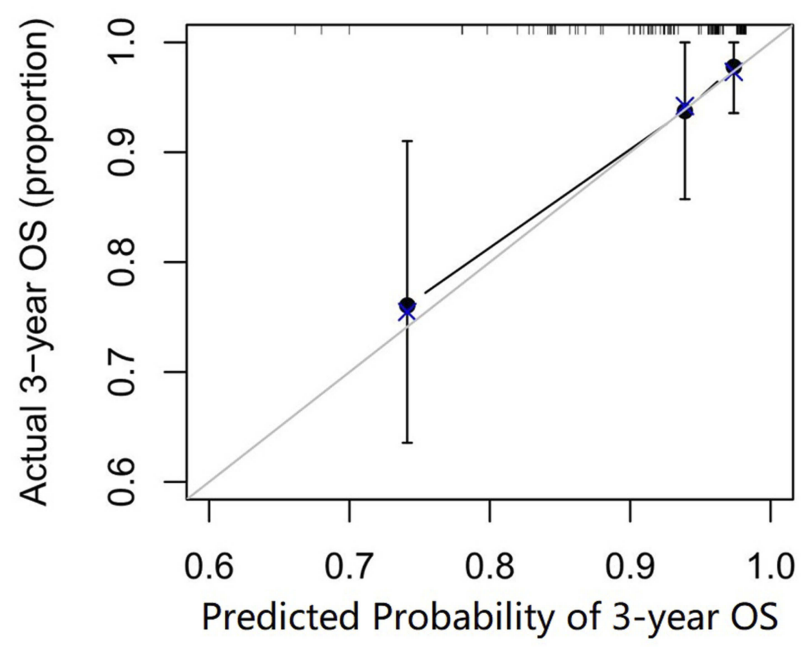

B

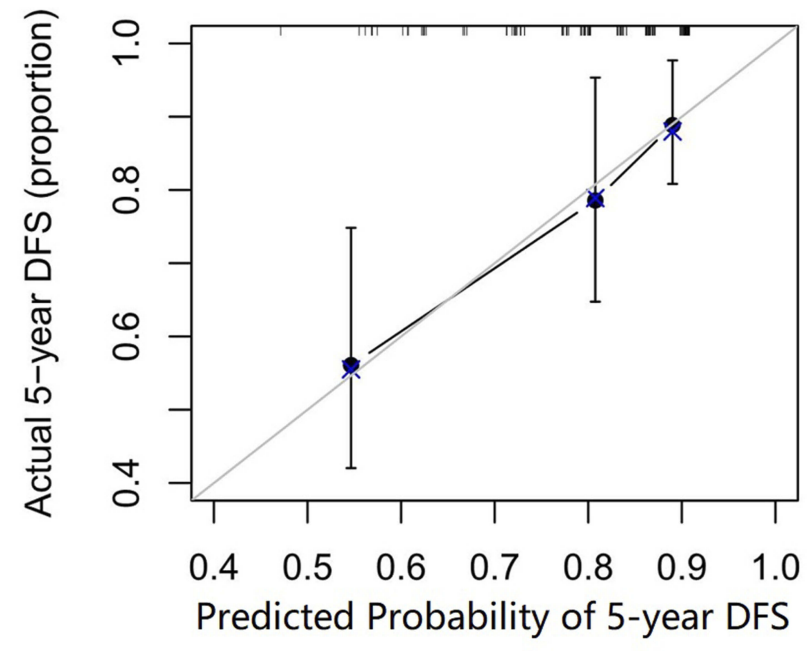

D

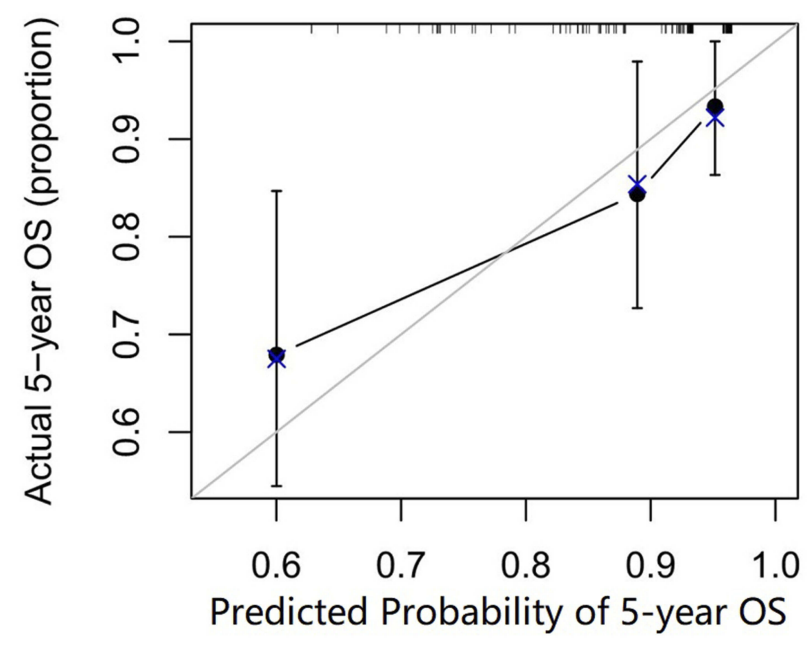

Figure 5 Calibration curves for predicting the OS and DFS for patients with triple-negative breast cancer in the validation group at (A, C) 3 years and (B, D) 5 years. Nomogram-predicted probability is plotted on the $x$-axis and the actual survival is plotted on the $y$-axis.

Abbreviations: DFS, disease-free survival; OS, overall survival.

Unfortunately, due to limited conditions, we did not test our tumor samples for TILs. We hope that this factor can be added to the prediction model in the future, which will certainly improve the accuracy of the model to a certain extent. Regarding the prediction factors based on the gene level, their wide clinical application in a short period of time was limited by the high testing cost and high requirements. Therefore, at present, we did not include these factors in the model.

Unlike previous nomograms of TNBC, we added common indicators of blood and biochemical examinations, owing to their frequent use in clinical practice. They are reliable, economical, and practical, greatly improving the operability of our prediction models. Moreover, our prediction model showed a high ability to predict the prognosis. We also developed two simple web pages based on our models.

This study had some limitations. Firstly, the nomogram was based on retrospective data obtained from a single medical institution. Secondly, we initiated with universality; thus, we did not study several predictive factors, such as tumor lymphocyte infiltration, BRCA1/2 mutation, other advanced technologies, and factors associated with high cost. Moreover, we did not study the specific classification of TNBC subtypes and their corresponding prognoses. In addition, adjuvant chemotherapy and radiotherapy were not included in this study. Further multi-center, prospective data collection, study on the classification of TNBC subtypes, 
A

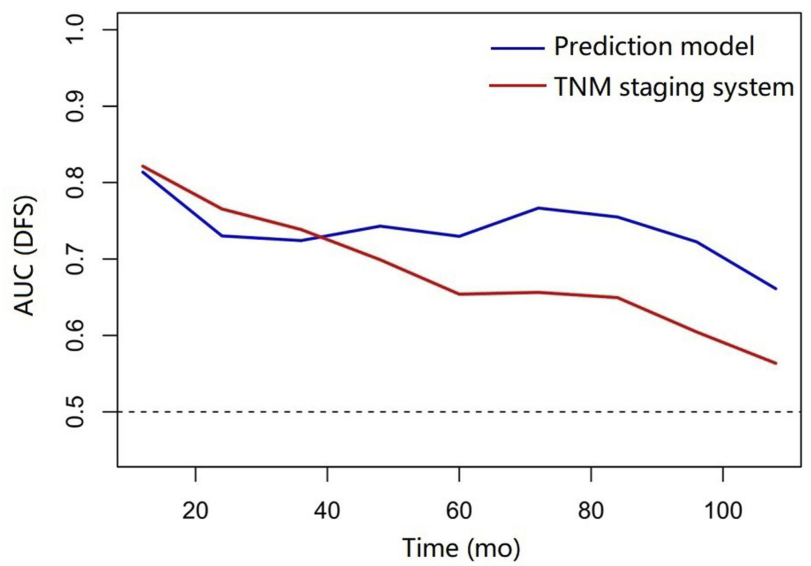

C

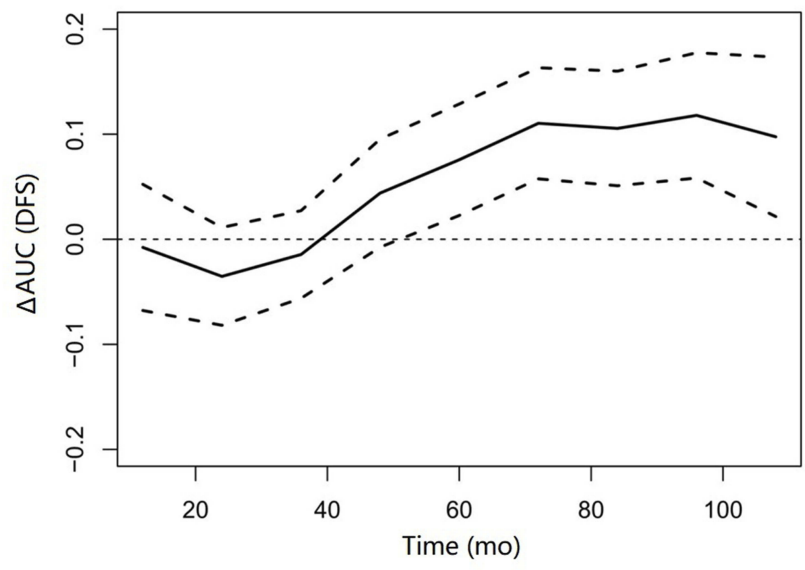

B

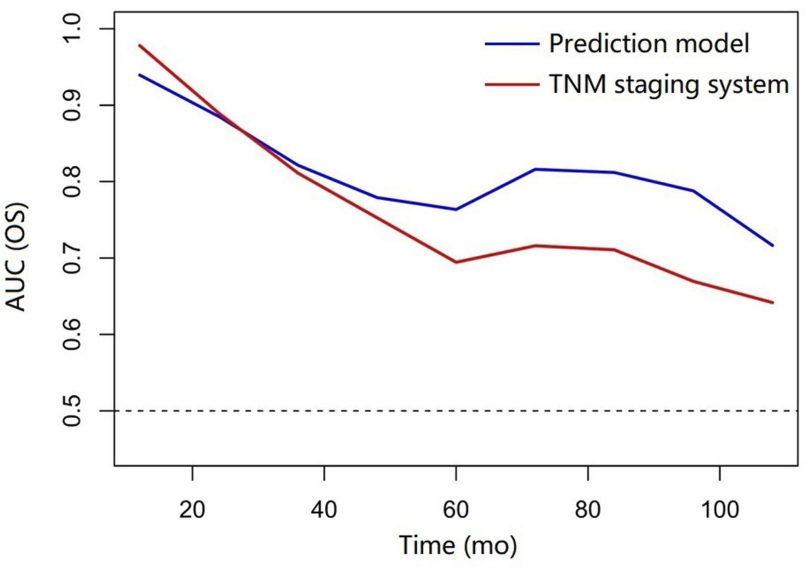

D

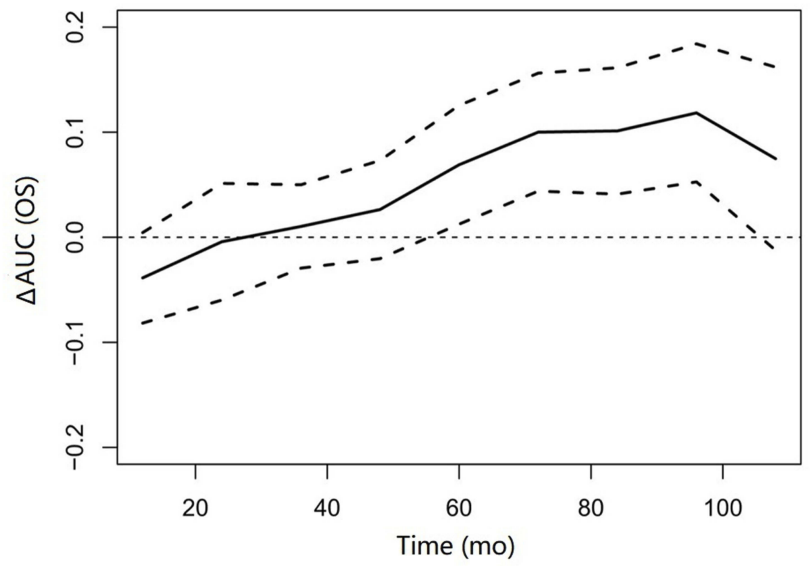

Figure 6 Time-dependent AUC of OS and DFS (A, B). Difference of AUC between the prediction models and the TNM staging system (C, D). Abbreviations: AUC, area under curve; OS, overall survival; DFS, disease-free survival; TNM, Tumor-Node-Metastasis.
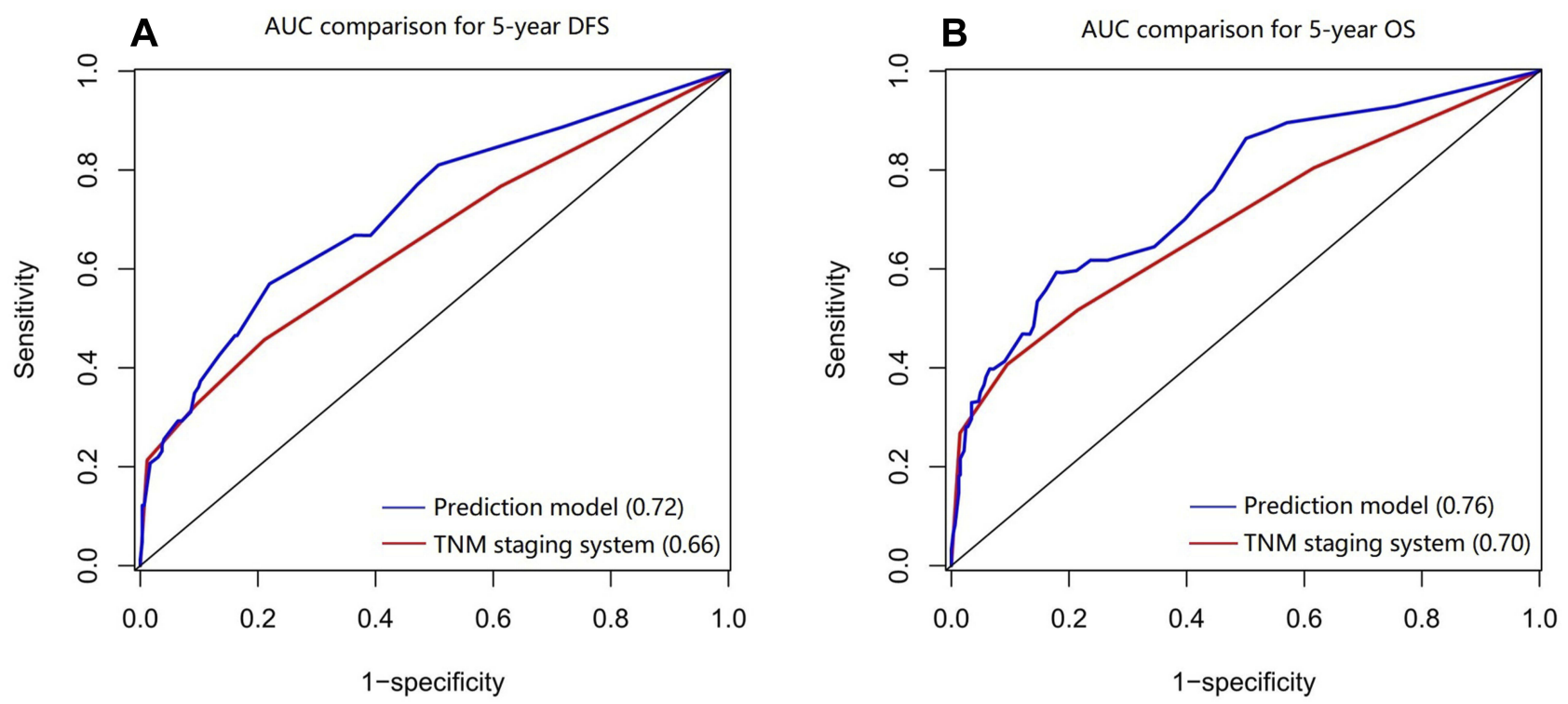

Figure 7 Five-year ROC curves.

Abbreviations: ROC, receiver operating characteristic; AUC, area under curve; DFS, disease-free survival; OS, overall survival; TNM, Tumor-Node-Metastasis. 
and integration of other factors may also contribute to the improvement of these models.

\section{Conclusion}

We have developed and validated universally applicable nomograms for predicting DFS and OS in patients with TNBC. These nomograms can provide relatively accurate prediction for the prognosis of patients with TNBC and identify those at different prognostic risks, thereby improving our alertness to strengthen the treatment or follow-up accordingly.

\section{Data Sharing Statement}

The dataset in this study is available by request from the corresponding author; for more information, please contact the corresponding author.

\section{Acknowledgements}

We thank Charlesworth Author Services for English language editing. This study was funded by Wenzhou Municipal Science and Technology Bureau Foundation (Y20170038) and Department of Health Foundation of Zhejiang Province (2019KY454).

\section{Ethics Approval and Informed Consent}

The present study was approved by the Medical Ethical Committee of the First Affiliated Hospital of Wenzhou Medical University and conformed to the provisions of the Declaration of Helsinki in 1995 (as revised in Edinburgh 2000). Owing to the retrospective nature of the study, a waiver for the requirement of individual informed consent was granted by the institutional ethics committee. We confirmed that the data were anonymized and analyzed with confidentiality.

\section{Disclosure}

The authors report no conflicts of interest in this work.

\section{References}

1. Bray F, Ferlay J, Soerjomataram I, Siegel RL, Torre LA, Jemal A. Global cancer statistics 2018: GLOBOCAN estimates of incidence and mortality worldwide for 36 cancers in 185 countries. CA Cancer J Clin. 2018;68(6):394-424. doi:10.3322/caac.v68.6

2. Chen W, Zheng R, Baade PD, et al. Cancer statistics in China, 2015. CA Cancer J Clin. 2016;66(2):115-132. doi:10.3322/caac.21338

3. Foulkes WD, Smith IE, Reis-Filho JS. Triple-negative breast cancer. $N$ Engl J Med. 2010;363(20):1938-1948. doi:10.1056/NEJMra100 1389
4. Criscitiello C, Azim HA, Schouten PC, Linn SC, Sotiriou C. Understanding the biology of triple-negative breast cancer. Ann Oncol. 2012;23(Suppl 6):vi13-vi18. doi:10.1093/annonc/mds188

5. Brewster AM, Chavez-MacGregor M, Brown P. Epidemiology, biology, and treatment of triple-negative breast cancer in women of African ancestry. Lancet Oncol. 2014;15(13):e625-e634. doi:10.10 16/S1470-2045(14)70364-X

6. Boyle P. Triple-negative breast cancer: epidemiological considerations and recommendations. Ann Oncol. 2012;23(suppl 6):vi7-vi12. doi:10.1093/annonc/mds187

7. Balachandran VP, Gonen M, Smith JJ, DeMatteo RP. Nomograms in oncology: more than meets the eye. Lancet Oncol. 2015;16(4):e173e180. doi:10.1016/S1470-2045(14)71116-7

8. Lin NU, Vanderplas A, Hughes ME, et al. Clinicopathologic features, patterns of recurrence, and survival among women with triple-negative breast cancer in the National Comprehensive Cancer Network. Cancer. 2012;118(22):5463-5472. doi:10.1002/cncr.27581

9. Montagna E, Maisonneuve P, Rotmensz N, et al. Heterogeneity of triple-negative breast cancer: histologic subtyping to inform the outcome. Clin Breast Cancer. 2013;13(1):31-39. doi:10.1016/j. clbc.2012.09.002

10. Dent R, Trudeau M, Pritchard KI, et al. Triple-negative breast cancer: clinical features and patterns of recurrence. Clin Cancer Res. 2007;13 (15 Pt 1):4429-4434. doi:10.1158/1078-0432.CCR-06-3045

11. Li CY, Wang P, Zhang S, Liu Y, Zhang J. Clincopathological features and prognosis of triple-negative breast cancer. Chin J Oncol. 2013;35 (6):173-181.

12. Yu F, Zhang XB, Zhang S, Liu JJ, Liu Y, Zhang J. Patterns and risk factors of recurrence in triple-negative breast cancer. Natl Med J China. 2014;94(28):2180-2183.

13. Diakos CI, Charles KA, McMillan DC, Clarke SJ. Cancer-related inflammation and treatment effectiveness. Lancet Oncol. 2014;15 (11):e493-e503. doi:10.1016/S1470-2045(14)70263-3

14. Jezequel P, Loussouarn D, Guerin-Charbonnel C, et al. Geneexpression molecular subtyping of triple-negative breast cancer tumours: importance of immune response. Breast Cancer Res. 2015;17:43. doi:10.1186/s13058-015-0550-y

15. Wang XJ, Xiang M, Tao YJ, et al. Application of preoperative neutrophil-to-lymphocyte ratio in the evaluation of prognosis in patients with triple-negative breast cancer. Oncol Prog. 2017;15(6).

16. Templeton AJ, McNamara MG, Seruga B, et al. Prognostic role of neutrophil-to-lymphocyte ratio in solid tumors: a systematic review and meta-analysis. $J$ Natl Cancer Inst. 2014;106(6):dju124. doi:10. 1093/jnci/dju124

17. Chen CZ, Lin CQ, Chen DL, et al. The effect and clinical significance of preoperative neutrophil-lymphocyte ratio on the prognosis of triple-negative breast cancer patients. Chin J General Surg. 2017;32(9):789-790.

18. Pistelli M, De Lisa M, Ballatore Z, et al. Pre-treatment neutrophil to lymphocyte ratio may be a useful tool in predicting survival in early triple negative breast cancer patients. BMC Cancer. 2015;15:195. doi:10.1186/s12885-015-1204-2

19. Hu B, Yang XR, Xu Y, et al. Systemic immune-inflammation index predicts prognosis of patients after curative resection for hepatocellular carcinoma. Clin Cancer Res. 2014;20(23):6212-6222. doi:10. 1158/1078-0432.CCR-14-0442

20. Aziz MH, Sideras K, Aziz NA, et al. The systemic-immuneinflammation index independently predicts survival and recurrence in resectable pancreatic cancer and its prognostic value depends on bilirubin levels: a retrospective multicenter cohort study. Ann Surg. 2019;270(1):139-146. doi:10.1097/SLA.000000 0000002660

21. Yang J, Guo X, Wang M, Ma X, Ye X, Lin P. Pre-treatment inflammatory indexes as predictors of survival and cetuximab efficacy in metastatic colorectal cancer patients with wild-type RAS. Sci Rep. 2017;7(1):17166. doi:10.1038/s41598-017-17130-6 
22. Tong YS, Tan J, Zhou XL, Song YQ, Song YJ. Systemic immune-inflammation index predicting chemoradiation resistance and poor outcome in patients with stage III non-small cell lung cancer. $J$ Transl Med. 2017;15(1):221. doi:10.1186/s12967-017-1326-1

23. Wang P, Yue W, Li W, et al. Systemic immune-inflammation index and ultrasonographic classification of breast imaging-reporting and data system predict outcomes of triple-negative breast cancer. Cancer Manag Res. 2019;11:813-819. doi:10.2147/CMAR.S185890

24. Guo W, Lu X, Liu Q, et al. Prognostic value of neutrophil-tolymphocyte ratio and platelet-to-lymphocyte ratio for breast cancer patients: an updated meta-analysis of 17079 individuals. Cancer Med. 2019;8(9):4135-4148. doi:10.1002/cam4.2281

25. Song W, Wang K, Zhong F, Fan YW, Peng L, Zou SB. Clinicopathological and prognostic significance of platelet-tolymphocyte ratio in patients with hepatocellular carcinoma. Oncotarget. 2016;7(49):81830. doi:10.18632/oncotarget.13244

26. Zou ZY, Liu HL, Ning N, Li SY, Du XH, Li R. Clinical significance of pre-operative neutrophil lymphocyte ratio and platelet lymphocyte ratio as prognostic factors for patients with colorectal cancer. Oncol Lett. 2016;11(3):2241-2248. doi:10.3892/ol.2016.4216

27. Liu J, Dai Y, Zhou F, et al. The prognostic role of preoperative serum albumin/globulin ratio in patients with bladder urothelial carcinoma undergoing radical cystectomy. Urol Oncol. 2016;34(11):484 e1-e8. doi:10.1016/j.urolonc.2016.05.024
28. Azab BN, Bhatt VR, Vonfrolio S, et al. Value of the pretreatment albumin to globulin ratio in predicting long-term mortality in breast cancer patients. Am J Surg. 2013;206(5):764-770. doi:10.1016/j. amjsurg.2013.03.007

29. Xuan Q, Yang Y, Ji H, et al. Combination of the preoperative albumin to globulin ratio and neutrophil to lymphocyte ratio as a novel prognostic factor in patients with triple negative breast cancer. Cancer Manag Res. 2019;11:5125-5131. doi:10.2147/ CMAR.S195324

30. Dai D, Chen B, Tang H, et al. Nomograms for predicting the prognostic value of pre-therapeutic CA15-3 and CEA serum levels in TNBC patients. PLoS One. 2016;11(8):e0161902. doi:10.1371/journal.pone.0161902

31. Adams S, Gray RJ, Demaria S, et al. Prognostic value of tumor-infiltrating lymphocytes in triple-negative breast cancers from two Phase III randomized adjuvant breast cancer trials: ECOG 2197 and ECOG 1199. J Clin Oncol. 2014;32(27):2959. doi:10.1200/ JCO.2013.55.0491

32. Dieci MV, Mathieu MC, Guarneri V, et al. Prognostic and predictive value of tumor infiltrating lymphocytes in two phase III randomized adjuvant breast cancer trials. Ann Oncol. 2015;8:1698-1704. doi:10. 1093/annonc/mdv239

\section{Publish your work in this journal}

Cancer Management and Research is an international, peer-reviewed open access journal focusing on cancer research and the optimal use of preventative and integrated treatment interventions to achieve improved outcomes, enhanced survival and quality of life for the cancer patient.
The manuscript management system is completely online and includes a very quick and fair peer-review system, which is all easy to use. Visit http://www.dovepress.com/testimonials.php to read real quotes from published authors. 